\title{
EFFECTS OF MACROPHAGE-ACTIVATING LIPOPEPTIDE-2 (MALP-2) ON THE VASCULARISATION OF IMPLANTED POLYURETHANE SCAFFOLDS SEEDED WITH MICROVASCULAR FRAGMENTS
}

\author{
C. Grässer ${ }^{1,2}$, C. Scheuer ${ }^{1,2}$, J. Parakenings ${ }^{1,2}$, T. Tschernig ${ }^{3}$, D. Eglin ${ }^{4}$, M.D. Menger ${ }^{1,2}$ and M.W. Laschke ${ }^{1,2, *}$ \\ ${ }^{1}$ Institute for Clinical \& Experimental Surgery, Saarland University, 66421 Homburg/Saar, Germany \\ ${ }^{2}$ Collaborative Research Partner Large Bone Defect Healing Program of AO Foundation \\ ${ }^{3}$ Institute for Anatomy, Saarland University, 66421 Homburg/Saar, Germany \\ ${ }^{4}$ AO Research Institute Davos, Clavadelerstrasse, 7270 Davos Platz, Switzerland
}

\begin{abstract}
The seeding of scaffolds with adipose tissue-derived microvascular fragments represents a promising strategy to establish a sufficient blood supply in tissue constructs. Herein, we analysed whether a single application of macrophage-activating lipopeptide-2 (MALP-2) at the implantation site further improves the early vascularisation of such microvessel-seeded constructs. Microvascular fragments were isolated from epididymal fat pads of C57BL/6 mice. The fragments were seeded on polyurethane scaffolds, which were implanted into mouse dorsal skinfold chambers exposed to MALP-2 or vehicle (control). The inflammatory host tissue response and the vascularisation of the scaffolds were analysed using intravital fluorescence microscopy, histology and immunohistochemistry. We found that the numbers of microvascular adherent leukocytes were significantly increased in MALP-2-treated chambers during the first 3 days after scaffold implantation when compared to controls. This temporary inflammation resulted in an improved vascularisation of the host tissue surrounding the implants, as indicated by a higher density of CD31-positive microvessels at day 14. However, the MALP-2-exposed scaffolds themselves presented with a lower functional microvessel density in their centre. In addition, in vitro analyses revealed that MALP-2 promotes apoptotic cell death of endothelial and perivascular cells in isolated microvascular fragments. Hence, despite the beneficial pro-angiogenic properties of MALP-2 at the implantation site, the herein evaluated approach may not be recommended to improve the vascularisation capacity of microvascular fragments in tissue engineering applications.
\end{abstract}

Keywords: Tissue engineering, MALP-2, microvascular fragments, angiogenesis, vascularisation, scaffold, polyurethane.

*Address for correspondence:

Matthias W. Laschke, M.D., Ph.D.

Institute for Clinical \& Experimental Surgery

Saarland University

D-66421 Homburg/Saar

Germany

Telephone number: +496841 1626554

Fax number: +4968411626553

Email: matthias.laschke@uks.eu

\section{Introduction}

The rapid establishment of a sufficient vascularisation is a crucial prerequisite for the survival of implanted tissue constructs (Novosel et al., 2011; Auger et al., 2013; Rouwkema and Khademhosseini, 2016). We and others have shown that this may be achieved by the seeding of tissue constructs with adipose tissue-derived microvascular fragments prior to their implantation (Hiscox et al., 2008; Laschke et al., 2012; Pilia et al., 2014).

Microvascular fragments are a mixture of arteriolar, capillary and venular vessel segments, which can be isolated by enzymatic digestion of fat tissue (Hoying et al., 1996; Shepherd et al., 2004; Nunes et al., 2010). The fragments release pro-angiogenic growth factors, such as vascular endothelial growth factor (VEGF) and basic fibroblast growth factor (bFGF) (Laschke et al., 2012; Pilia et al., 2014). In addition, they are a rich source of mesenchymal stem cells and endothelial progenitor cells (Laschke et al., 2012; McDaniel et al., 2014). After their incorporation into scaffolds they reassemble to functional microvascular networks during the first $6 \mathrm{~d}$ after implantation (Laschke et al., 2012). In the present study, we speculated that this initial period, which is characterised by critical hypoxia within the implants, may be further shortened by local application of macrophage-activating lipopeptide-2 (MALP-2) at the implantation site.

MALP-2 is a toll-like receptor (TLR) 2/6 agonist originating from mycoplasma species, which is a potent stimulator of the immune system (Mühlradt et al., 1997). Accordingly, the compound has been suggested as a suitable mucosal adjuvant for vaccination (Rharbaoui et al., 2002; Lührmann et al., 2005) and inducer of lipopolysaccharide cross tolerance (Deiters et al., 2003). More recent studies demonstrate that MALP-2 promotes angiogenesis by up-regulation of granulocyte-macrophage colony-stimulating factor (GM-CSF) (Grote et al., 2010) and improves re-endothelialisation after vascular injury (Grote et al., 2013a). Importantly, the angiogenic potential of MALP-2 has been shown in vitro and in vivo to be comparable to that of VEGF and bFGF (Grote et $a l ., 2010)$. In addition, we found that the early vascular ingrowth into porous polyethylene (Medpor ${ }^{\circledR}$ ) scaffolds is markedly improved by local single-shot application of MALP-2 at the implantation site (Laschke et al., 2014a). In contrast to the coating of scaffolds with angiogenic growth factor delivery systems, the local single-shot application of MALP-2 at the implantation site may be particularly attractive for clinical purposes, because it does not involve complex biological implant modifications. Moreover, 
the stimulation of angiogenesis by MALP-2 is partly mediated by a short-term accumulation and activation of immune cells (Laschke et al., 2014a), which may increase bacterial clearance and, thus, reduce the risk of implant infections. Finally, MALP-2 has already been proven to enhance wound healing (Niebuhr et al., 2008) and to suppress tumour growth (Schmidt et al., 2007) in clinical phase I/II studies without inducing systemic side effects. However, the effects of MALP-2 on the vascularisation of scaffolds seeded with microvascular fragments have not been analysed so far.

Therefore, we herein seeded polyurethane scaffolds with microvascular fragments, which were implanted into MALP-2- or vehicle-treated mouse dorsal skinfold chambers. The angiogenic and inflammatory host tissue response to the implants was analysed by means of repetitive intravital fluorescence microscopy, histology and immunohistochemistry over an observation period of $14 \mathrm{~d}$.

\section{Materials and Methods}

\section{Animals}

This study was approved by the local governmental animal care committee. All animal experiments were conducted in accordance with the German legislation on protection of animals and the NIH Guidelines for the Care and Use of Laboratory Animals (NIH Publication \#85-23 Rev. 1985).

We used male C57BL/6 mice with an average age of 8 months and a body weight of $30-35 \mathrm{~g}$ as donors for the isolation of microvascular fragments to guarantee large epididymal fat pads and, thus, sufficient amounts of microvascular fragments for the seeding of the scaffolds. Dorsal skinfold chambers were implanted into C57BL/6 mice with an average age of 3-4 months and a body weight of 22-26 g (Institute for Clinical \& Experimental Surgery, Saarland University, Homburg/Saar, Germany), to guarantee optimal implantation conditions. The animals were housed one per cage and had free access to tap water and standard pellet food throughout the experiments (Altromin, Lage, Germany).

\section{Isolation of microvascular fragments and seeding of scaffolds}

For the isolation of microvascular fragments, donor mice were anaesthetised by intraperitoneal (i.p.) injection of ketamine $(75 \mathrm{mg} / \mathrm{kg}$ body weight; Pharmacia $\mathrm{GmbH}$, Erlangen, Germany) and xylazine $(25 \mathrm{mg} / \mathrm{kg}$ body weight; Rompun, Bayer, Leverkusen, Germany). Subsequently, the two epidydimal fat pads were carefully excised by means of a microscissors and enzymatically digested, as described previously in detail (Laschke et al., 2012). Directly after the isolation procedure, we assessed the number of microvascular fragments from 2 donor animals by means of a Leica microscope with digital camera (Leica DM IL; Leica Microsystems, Wetzlar, Germany) and computer-assisted analysis system (Leica Application Suite 4.8; Leica Microsystems). Our analysis revealed that $16,165 \pm 545$ microvascular fragments per donor animal could be isolated. This was also the number of fragments which was seeded per scaffold.

The microvascular fragments were seeded on porous nano-size hydroxyapatite particles/poly(ester-urethane) composite scaffolds, which have originally been generated for bone tissue engineering applications (Laschke et al., 2010). These scaffolds exhibit a size of $\sim 3 \times 3 \times 1 \mathrm{~mm}$ and interconnected macropores of $\sim 220 \mu \mathrm{m}$. Thus, they ideally support vascular ingrowth and can be used as standardised matrices for the evaluation of different vascularisation approaches (Laschke et al., 2013).

For the seeding of the scaffolds with microvascular fragments, they were fixed in the lumen of a modified $1 \mathrm{~mL}$ syringe (BD Plastipak; BD Biosciences, Heidelberg, Germany), as described previously in detail (Laschke et al., 2014b). The tip of the syringe was filled with $20 \mu \mathrm{L}$ PBS containing the microvascular fragments. Negative and positive pressure was alternately induced three to five times in the syringe so that the microvascular fragments passed the scaffolds from both sides and were finally trapped in the scaffold pores (Laschke et al., 2014b). Histological analyses of freshly seeded scaffolds revealed that the microvascular fragments were randomly distributed on the scaffolds' surface and in the centre pores (Fig. 1a).

\section{Dorsal skinfold chamber model}

The effect of MALP-2 on the vascularisation of microvesselseeded scaffolds was analysed in the dorsal skinfold chamber model by means of intravital fluorescence microscopy (Fig. 1b). A detailed description of the chamber preparation is given by Laschke et al. (2011). The animals were allowed to recover from the preparation for $48 \mathrm{~h}$ prior to the treatment of the chamber tissue with MALP-2 and the implantation of the scaffolds.

MALP-2 (MALP-2 Research, Braunschweig, Germany) was kept frozen at $-20{ }^{\circ} \mathrm{C}$ as a stock solution of $1 \mathrm{mg} / \mathrm{mL}$ in $30 \%$ isopropanol. For the experiments the stock solution was diluted with $0.9 \% \mathrm{NaCl}$ to reach a final dose of $0.5 \mu \mathrm{g}$ MALP-2 dissolved in $150 \mu \mathrm{L}(3.3 \mu \mathrm{g} / \mathrm{mL})$. This dose has previously been shown to induce a strong angiogenic host tissue reaction to implanted scaffolds in the dorsal skinfold chamber (Laschke et al., 2014a). After removal of the cover glass, MALP-2 or vehicle was topically applied onto the exposed striated muscle tissue of the chamber for $30 \mathrm{~min}$. Subsequently, one scaffold seeded with microvascular fragments was centrally placed into the MALP-2 or vehicle without prior rinsing of the chamber tissue (Fig. 1c), so that the solutions could also be absorbed into the pores of the implant. After sealing the observation window with a new cover class, intravital fluorescence microscopy was repetitively performed over two weeks, as described previously in detail (Laschke et al., 2014b) (Fig. 1d,e). For this purpose, $0.05 \mathrm{~mL} 5 \%$ fluorescein isothiocyanate (FITC)-labelled dextran 150,000 (Sigma-Aldrich, Taufkirchen, Germany) and $0.05 \mathrm{~mL}$ $0.1 \%$ rhodamine $6 \mathrm{G}$ (Sigma-Aldrich) were injected i.v. via the retrobulbary space of the anaesthetised mice. The two vital dyes were administered for contrast enhancement by staining of blood plasma (Debergh et al., 2015) and for direct in vivo staining of leukocytes (Baatz et al., 1995; 

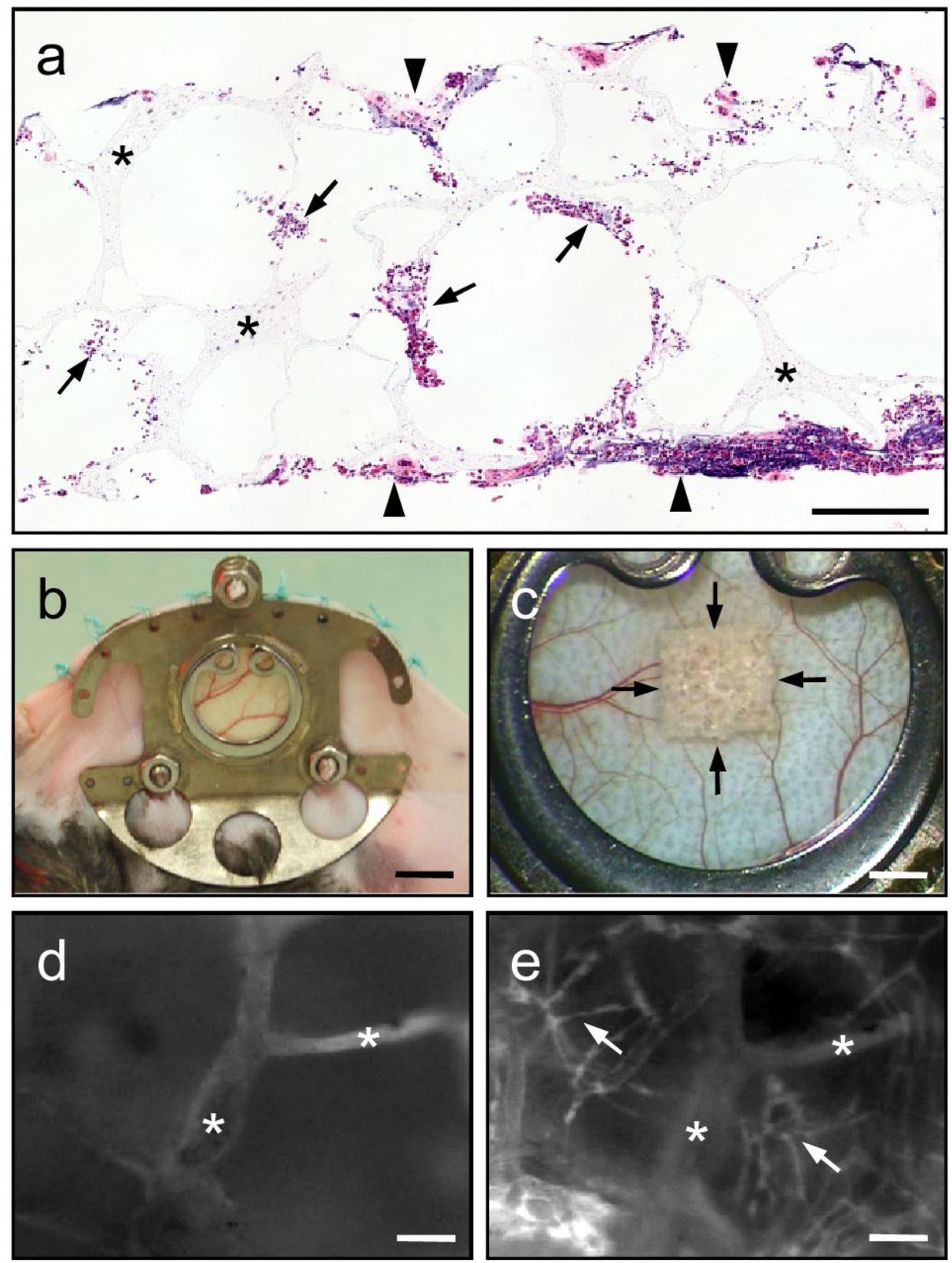

Fig. 1. (a) HE-stained section of a polyurethane scaffold (strands marked by asterisks) directly after the seeding with microvascular fragments. The microvascular fragments are randomly distributed on the scaffold's surface (arrowheads) and in the centre pores (arrows). (b) Dorsal skinfold chamber (chamber weight $\sim 2 \mathrm{~g}$ ) on the back of a C57BL/6 mouse. (c) Overview of the observation window of a chamber directly after implantation of a microvessel-seeded polyurethane scaffold (borders marked by arrows). (d,e) Intravital fluorescence microscopy of a microvessel-seeded polyurethane scaffold directly (d) as well as at day 14 (e) after implantation into the dorsal skinfold chamber. The scaffold (polyurethane strands marked by asterisks) initially lacks a vascularisation (d). Due to the rapid interconnection of the microvascular fragments the implant finally exhibits a dense network of blood-perfused microvessels (e, arrows). Bluelight epi-illumination with contrast enhancement by $5 \%$ FITC-labelled dextran 150,000 i.v.. Scale bars: a $=200 \mu$ m; $\mathrm{b}=6.3 \mathrm{~mm} ; \mathrm{c}=1.6 \mathrm{~mm} ; \mathrm{d}, \mathrm{e}=65 \mu \mathrm{m}$.

Herr et al., 2015). The microscopic images were recorded by a charge-coupled device video camera (FK6990; Pieper, Schwerte, Germany) and transferred to a DVD system for off-line analysis.

\section{Microcirculatory analysis}

The microscopic images were analysed with CapImage (Zeintl, Heidelberg, Germany). The vascularisation of the scaffolds was assessed at a magnification of $\times 460$ in 8 different microvascular regions of interest (ROIs) within the implants. Perfused ROIs (given in \% of all analysed ROIs) were defined as areas, which exhibited either newly developed red blood cell (RBC)-perfused microvessels or reperfused microvascular fragments. In addition, the functional microvessel density, i.e. the length of all RBC-perfused blood vessels per ROI, was assessed 
and is given in $\mathrm{cm} / \mathrm{cm}^{2}$ (Laschke et al., 2012). Finally, we measured the diameter ( $d$; given in $\mu \mathrm{m}$ ) and the centreline $\mathrm{RBC}$ velocity ( $v$; given in $\mu \mathrm{m} / \mathrm{s}$ ) of 25 randomly selected microvessels in the centre of the implants, which already exhibited a blood perfusion at the time point of analysis. The wall shear rate $\left(y\right.$; given in $\left.\mathrm{s}^{-1}\right)$ of these vessels was then calculated based on the Newtonian definition: $y=8$ * v/d (Kremer et al., 2008).

Leukocyte-endothelial cell interaction was analysed at a $\times 460$ magnification in 4 different microvascular regions of interest (ROIs) next to the implants. For this purpose, rhodamine 6G-stained rolling leukocytes and adherent leukocytes were quantified in 1-3 postcapillary or collecting venules per ROI. Rolling leukocytes were defined as cells moving with a velocity less than two-fifth of the centreline velocity. They are expressed as the number of cells per minute that passed a reference point within the microvessel (Hoffmann et al., 2004). Adherent leukocytes were defined as cells that did not move or detach from the endothelial lining within a $20 \mathrm{~s}$ observation period. They are expressed as number of cells per $\mathrm{mm}^{2}$ of venular endothelial surface assuming cylindrical vessel geometry (Hoffmann et al., 2004). Finally, the diameter, centreline $\mathrm{RBC}$ velocity and wall shear rate of the venules were determined.

\section{Experimental protocol}

Microvascular fragments were isolated from the epididymal fat pads of 6 male wild-type C57BL/6 mice. Directly after the isolation procedure, we assessed the number of microvascular fragments from 2 donor animals by brightfield microscopy. The microvascular fragments of the other 4 animals were seeded on 4 scaffolds, which were then directly processed for the histological assessment of the seeding quality without in vivo implantation.

Microvascular fragments from the epididymal fat pads of 14 C57BL/6 donor mice were seeded on 14 scaffolds. Subsequently, the scaffolds were implanted into the dorsal skinfold chambers of 14 C57BL/6 recipient mice, which were exposed to MALP-2 ( $n=7$ biological replicates) or vehicle (control; $n=7$ biological replicates). Intravital fluorescence microscopy was performed immediately after implantation (day 0) as well as at day 3, 6, 10 and 14. At the end of the in vivo experiments, the animals were sacrificed with an overdose of the anaesthetics and the dorsal skinfold chamber preparations were excised for histological and immunohistochemical analyses.

In an additional set of in vitro experiments, microvascular fragments were isolated from the epididymal fat pads of 10 C57BL/6 donor mice and cultured for $24 \mathrm{~h}$ in 24-well plates containing $500 \mu \mathrm{L}$ endothelial cell growth medium MV (EC medium; PromoCell, Heidelberg, Germany) supplemented with $3.3 \mu \mathrm{g} / \mathrm{mL}$ MALP-2 or vehicle. Subsequently, the fragments were embedded in $1 \%$ agarose (Sigma-Aldrich) and further processed for immunohistochemical analyses.

\section{Histology and immunohistochemistry}

Formalin-fixed specimens of freshly seeded scaffolds and dorsal skinfold preparations with scaffold implants were embedded in paraffin and cut into $3 \mu \mathrm{m}$-thick sections.
Haematoxylin and eosin (HE) staining of individual sections was performed according to standard procedures.

For immunohistochemical analyses, paraffin-embedded $3 \mu \mathrm{m}$-thick sections were stained with a monoclonal ratanti-mouse antibody against CD31 (6 $\mu \mathrm{g} / \mathrm{mL}$; Dianova $\mathrm{GmbH}$, Hamburg, Germany) and with a polyclonal rabbit-anti-mouse antibody against cleaved caspase-3 $(0.63 \mu \mathrm{g} / \mathrm{mL}$; New England Biolabs, Frankfurt am Main, Germany). A goat-anti-rat IgG-Cy3 antibody $(4.5 \mu \mathrm{g} / \mathrm{mL}$; Dianova $\mathrm{GmbH}$ ), a goat-anti-rat IgG-Alexa488 antibody $(20 \mu \mathrm{g} / \mathrm{mL}$; Invitrogen, Eugene, OR, USA) and a goat-antirabbit IgG-biotinylated antibody (ready-to-use; Abcam) served as secondary antibodies. The biotinylated antibody was detected by Cy3-labeled-streptavidin $(50 \mu \mathrm{g} / \mathrm{mL}$; Invitrogen). On each section, cell nuclei were stained with Hoechst 33342 (2 $\mu \mathrm{g} / \mathrm{mL}$; Sigma-Aldrich) to merge the images exactly. The sections were examined using a BX60 microscope (Olympus, Hamburg, Germany). Quantitative analyses of the sections included the determination of the microvessel density (CD31-stained cells; given in $\mathrm{mm}^{-2}$ ) in the border and centre of the implanted scaffolds. Moreover, we assessed the fraction (given in \%) of cleaved caspase-3positive endothelial and perivascular cells in microvascular fragments, which were cultured for $24 \mathrm{~h}$ in EC medium supplemented with MALP-2 or vehicle.

\section{Statistics}

After testing the data for normal distribution and equal variance, differences between the two groups were analysed by the unpaired Student's $t$-test. To test for time effects in the individual groups, ANOVA for repeated measures was applied. This was followed by the StudentNewman-Keuls test, including the correction of the alpha error according to Bonferroni probabilities to compensate for multiple comparisons (SigmaPlot 11.0; Systat Software Inc., San Jose, CA, USA). All values are expressed as means \pm SEM. Statistical significance was accepted for a value of $p<0.05$.

\section{Results}

\section{Inflammatory tissue response to MALP-2}

To assess the inflammatory response to locally applied MALP-2, we first analysed the leukocyte-endothelial cell interaction in postcapillary and collecting venules of the chamber tissue by means of intravital fluorescence microscopy. Throughout the observation period of $14 \mathrm{~d}$, the analysed vessels exhibited a comparable diameter, centreline $\mathrm{RBC}$ velocity and shear rate without significant differences between MALP-2-treated and vehicle-treated chambers (Table 1).

Stimulation of the chamber tissue with MALP-2 did not affect the number of rolling leukocytes, which ranged between 22-33 $\mathrm{min}^{-1}$ in vehicle-treated and MALP-2treated venules without significant differences (Fig. 2c). In contrast, MALP-2 markedly increased the number of adherent leukocytes during the first $3 \mathrm{~d}$ after application when compared to vehicle-treated controls (Figs. 2a,b,d). The number of adherent leukocytes was even higher 
Table 1. Micro-haemodynamic parameters of venules in close vicinity of microvessel-seeded scaffolds

\begin{tabular}{|l|c|c|c|c|c|}
\hline & $\mathbf{0 ~ d}$ & $\mathbf{3 ~ d}$ & $\mathbf{6 ~ d}$ & $\mathbf{1 0 ~ d}$ & $\mathbf{1 4 ~ d}$ \\
\hline Diameter $[\mu \mathrm{m}]:$ & & & & & \\
\hline Control & $43.4 \pm 1.7$ & $41.7 \pm 2.7$ & $43.6 \pm 2.7$ & $45.7 \pm 8.2$ & $36.4 \pm 1.3$ \\
\hline MALP-2 & $41.9 \pm 1.6$ & $40.6 \pm 1.1$ & $41.8 \pm 1.7$ & $43.7 \pm 1.5$ & $39.4 \pm 1.2$ \\
\hline Centreline RBC velocity $[\mu \mathrm{m} / \mathrm{s}]:$ & & & & & \\
\hline Control & $702.9 \pm 68.4$ & $711.6 \pm 132.7$ & $764.3 \pm 129.2$ & $704.8 \pm 102.7$ & $588.1 \pm 37.5$ \\
\hline MALP-2 & $597.2 \pm 70.0$ & $511.2 \pm 56.6$ & $644.7 \pm 100.9$ & $481.8 \pm 117.2$ & $720.5 \pm 62.1$ \\
\hline Wall shear rate $\left[\mathrm{s}^{-1}\right]:$ & & & & \\
\hline Control & & & & & \\
\hline MALP-2 & $133.1 \pm 18.1$ & $135.3 \pm 23.1$ & $140.1 \pm 21.3$ & $130.7 \pm 22.9$ & $130.2 \pm 9.4$ \\
\hline
\end{tabular}

Diameter $(\mu \mathrm{m})$, centreline RBC velocity $(\mu \mathrm{m} / \mathrm{s})$ and wall shear rate $\left(\mathrm{s}^{-1}\right)$ of postcapillary and collecting venules in close vicinity of microvessel-seeded scaffolds directly $(0 \mathrm{~d})$ as well as 3, 6, 10 and $14 \mathrm{~d}$ after implantation into the dorsal skinfold chambers, as assessed by intravital fluorescence microscopy and computer-assisted off-line analysis. The chamber tissue was exposed to MALP-2 $(n=7)$ or vehicle (control; $n=7) 30$ min before implantation of the scaffolds. Mean \pm SEM.

than $600 \mathrm{~mm}^{-2}$ at the day of scaffold implantation, which indicates strong tissue inflammation in the dorsal skinfold chamber model (Rücker et al., 2006). However, this inflammatory tissue response was only of short duration. Thereafter, numbers of adherent leukocytes returned to physiological levels $<200 \mathrm{~mm}^{-2}$, which are typical for non-inflamed chamber tissue (Rücker et al., 2006; Laschke et al., 2010).

\section{Vascularisation of implanted scaffolds}

To assess the effect of MALP-2 on the vascularisation capacity of the microvascular fragments within the polyurethane scaffolds, we analysed the number of perfused ROIs and the functional microvessel density in central surface areas of the implants. In previous studies, we implanted scaffolds seeded with microvascular fragments from transgenic green fluorescent protein (GFP)-positive donor mice in the dorsal skinfold chamber of GFP-negative wild-type recipient animals (Laschke et al., 2012; Laschke et al., 2015). By this, we could demonstrate that the vascularisation in these central surface areas of the implants originates nearly exclusively from the microvascular fragments and not from ingrowing microvessels of the surrounding host tissue.

In vehicle-treated and MALP-2-treated chambers first perfused microvessels could be detected in the scaffolds' centre at day 6 after implantation (Fig. 3c,d). This indicates that, to this time point, the microvascular fragments had developed interconnections to the blood-supplying microvessels of the implantation site. Throughout the further time course of the experiment, an increasing number of vascular sprouts grew out of the perfused microvessels, finally resulting in the formation of new microvascular networks within the implanted scaffolds. Unexpectedly, this process was markedly altered in MALP-2-treated chambers. The scaffolds exposed to MALP-2 exhibited a delayed perfusion, as indicated by a significantly reduced number of perfused ROIs at day 10 when compared to vehicle-treated controls (Fig. 3c). In addition, the functional microvessel density within these scaffolds was significantly lower at day 10 and 14 after implantation (Fig. $3 \mathbf{a}, \mathbf{b}, \mathbf{d})$.

Additional micro-haemodynamic analyses revealed that at day 6 the re-perfused microvascular fragments exhibited comparable diameters of $\sim 60 \mu \mathrm{m}$ within the scaffolds of the two groups (Fig. 4c). During the following days, the vessel diameters progressively decreased until day 14 as a typical sign of vessel stabilisation and vascular remodelling. Of interest, this decrease was less pronounced in the MALP-2-treated chambers (Fig. 4a-c). Moreover, at day 14 centreline RBC velocities and shear rates were significantly lower in the newly formed microvascular networks of scaffolds within MALP-2-treated chambers when compared to controls (Fig. 4d,e).

\section{Incorporation of scaffolds into the surrounding host tissue}

Histological analyses of the dorsal skinfold chamber preparations at day 14 showed that the scaffolds were surrounded by a newly formed granulation tissue, which also grew into the pores of the implants (Fig. 5a,b). This ingrowth was less pronounced in the scaffolds of MALP2-treated chambers, indicating a deteriorated incorporation of the implants when compared to controls.

Additional immunohistochemical analyses revealed a significantly higher density of CD31-positive microvessels at the border of scaffolds in MALP-2-treated chambers (Fig. 5c-e). This shows that the treatment with MALP2 stimulated angiogenesis in the granulation tissue in direct vicinity to the implants. However, the number of CD31-positive microvessels was significantly lower in the scaffolds' centre when compared to controls (Figs. $5 \mathbf{c}-\mathbf{e})$. This finding is in line with our intravital fluorescent microscopic results, indicating an impaired vascularisation capacity of microvascular fragments exposed to MALP-2.

\section{Effect of MALP-2 on cell viability in microvascular fragments}

Microvascular fragments are free of adipocytes, because they are destroyed during the enzymatic isolation procedure 

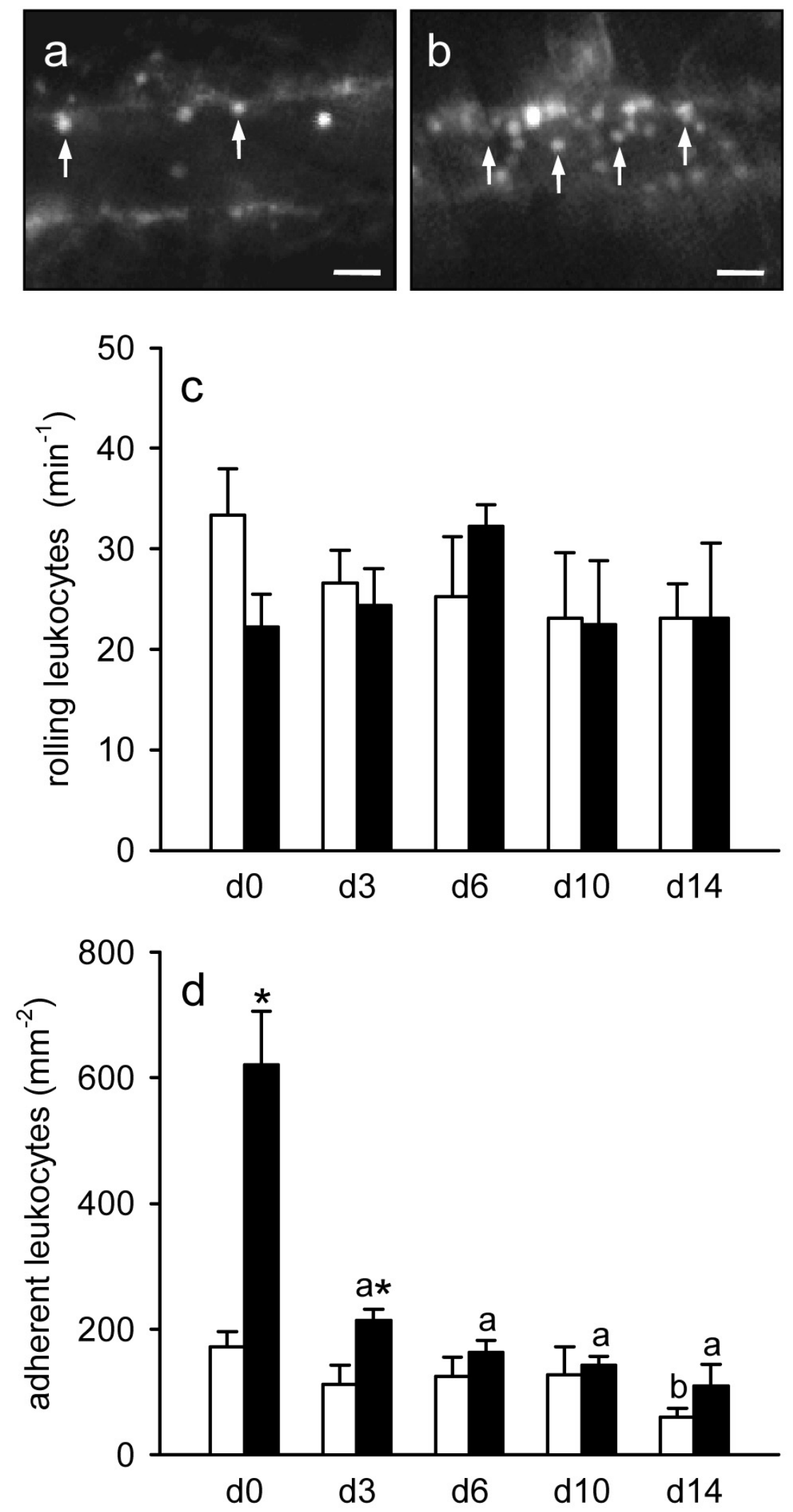

Fig. 2. (a,b) Intravital fluorescence microscopy of venules within the dorsal skinfold chamber directly after the implantation of microvessel-seeded polyurethane scaffolds. The chamber tissue was treated with vehicle (control, a) or MALP-2 (b) 30 min before scaffold implantation. Note that the venule exposed to MALP-2 (b) exhibits a higher number of adherent leukocytes (arrows) when compared to the vehicle-treated control (a, arrows). Green-light epi-illumination with visualisation of leukocytes by rhodamine 6G i.v.. Scale bars: $22 \mu \mathrm{m}$. (c,d) Numbers of rolling leukocytes $\left(\mathrm{min}^{-1}\right)$ (c) and adherent leukocytes $\left(\mathrm{mm}^{-2}\right)(\mathbf{d})$ in venules within vehicle-treated (white bars, $\left.n=7\right)$ and MALP-2-treated (black bars, $n=7$ ) dorsal skinfold chambers directly (d0) as well as at day 3, 6, 10 and 14 after scaffold implantation, as assessed by intravital fluorescence microscopy and computer-assisted off-line analysis. Means \pm SEM. ${ }^{\mathrm{a}} p<0.05 v s$. $\mathrm{d} 0 ;{ }^{\mathrm{b}} p<0.05$ vs. $\mathrm{d} 0, \mathrm{~d} 3, \mathrm{~d} 6$ and $\mathrm{d} 10 ;{ }^{*} p<0.05$ vs. control. 

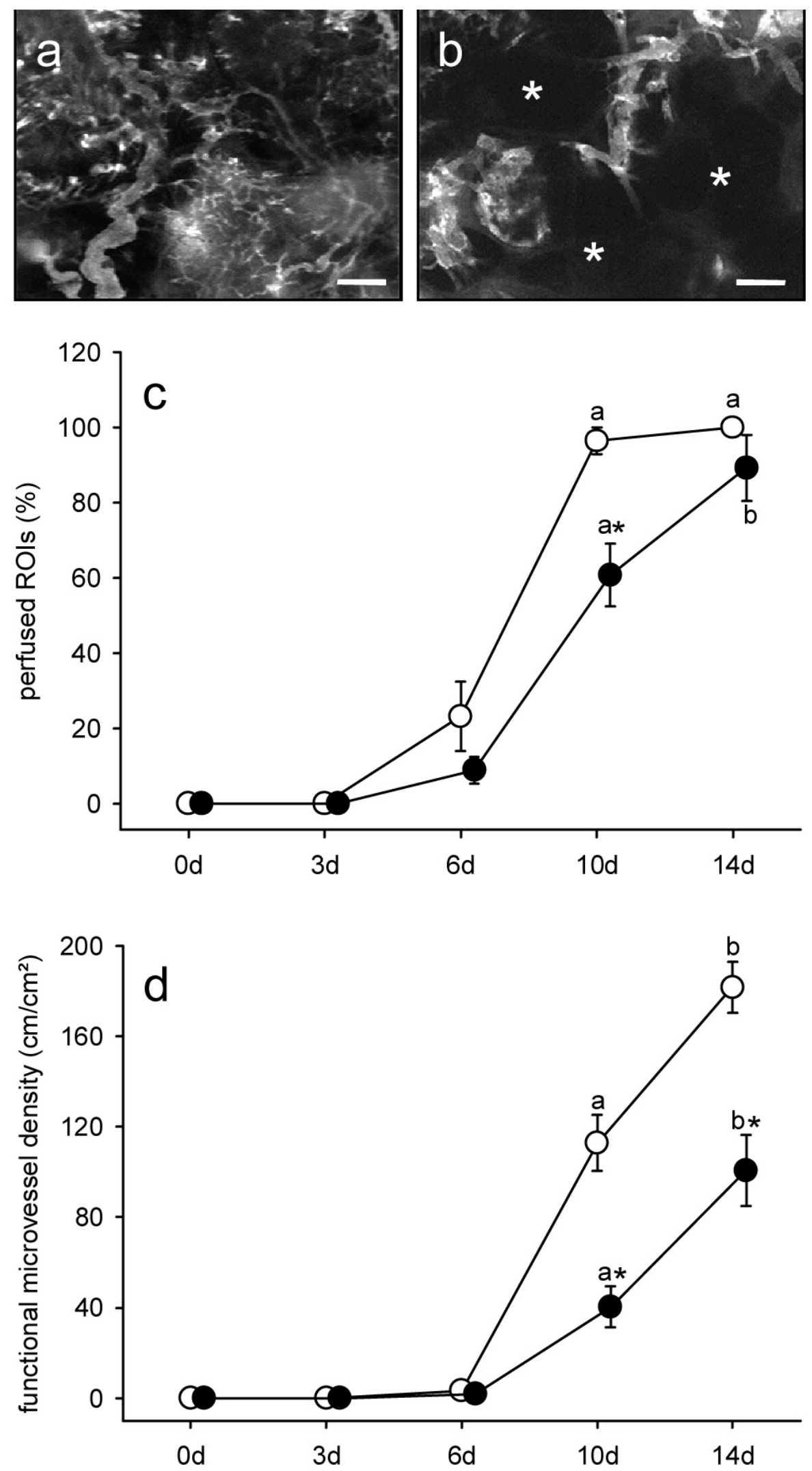

Fig. 3. (a,b) Intravital fluorescence microscopy of microvessel-seeded polyurethane scaffolds at day 14 after implantation into a vehicle-treated (a) and a MALP-2-treated (b) dorsal skinfold chamber. Note that the scaffold in the vehicle-treated chamber contains a dense network of blood-perfused microvessels (a), whereas the scaffold in the MALP-2-treated chamber still exhibits large avascular areas (b, asterisks). Blue-light epi-illumination with contrast enhancement by $5 \%$ FITC-labelled dextran 150,000 i.v.. Scale bars: $230 \mu \mathrm{m}$. (c, d) Perfused ROIs (\%) (c) and functional microvessel density $\left(\mathrm{cm} / \mathrm{cm}^{2}\right)(\mathbf{d})$ of microvessel-seeded polyurethane scaffolds directly (d0) as well as at day 3, 6, 10 and 14 after implantation into vehicle-treated (white circles, $n=7$ ) and MALP-2-treated (black circles, $n=7$ ) dorsal skinfold chambers of C57BL/6 mice, as assessed by intravital fluorescence microscopy and computer-assisted off-line analysis. Means \pm SEM. ${ }^{\mathrm{a}} p<0.05$ vs. d0; ${ }^{\mathrm{b}} p<0.05$ vs. d0, d3, d6 and d10; $* p<0.05$ vs. control. 

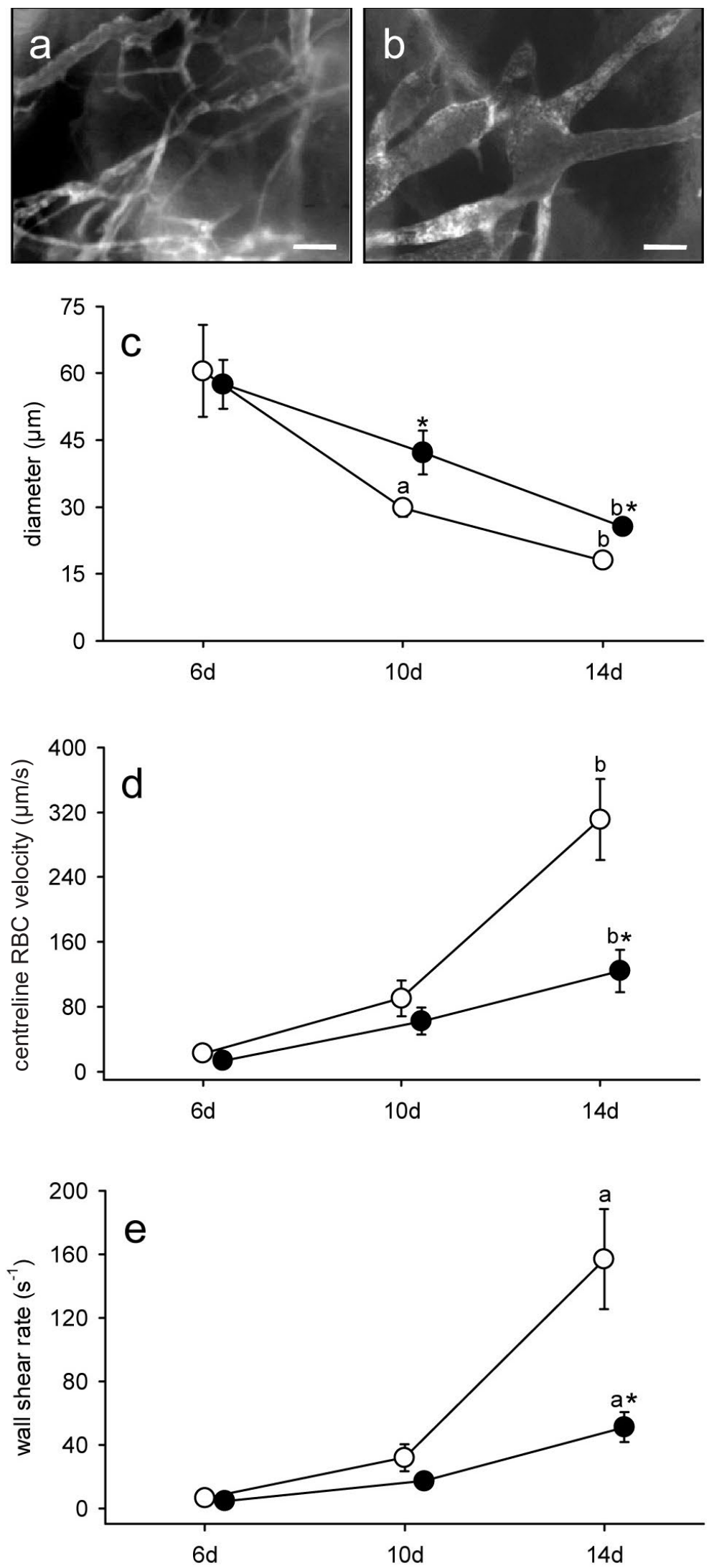

Fig. 4. (a,b) Intravital fluorescence microscopy of microvessels within polyurethane scaffolds at day 14 after implantation into a vehicle-treated (a) and a MALP-2-treated (b) dorsal skinfold chamber. Note that the microvessels in (a) exhibit smaller diameters when compared to those in (b). Blue-light epi-illumination with contrast enhancement by $5 \%$ FITClabelled dextran 150,000 i.v.. Scale bars: $65 \mu \mathrm{m}$. (c-e) Diameter $(\mu \mathrm{m})(\mathbf{c})$, centreline RBC velocity $(\mu \mathrm{m} / \mathrm{s})(\mathrm{d})$ and wall shear rate $\left(\mathrm{s}^{-1}\right)$ (e) of microvessels in polyurethane scaffolds at day 6, 10 and 14 after implantation into vehicle-treated (control; white circles, $n=7$ ) and MALP-2-treated (black circles, $n=7$ ) dorsal skinfold chambers, as assessed by intravital fluorescence microscopy and computer-assisted off-line analysis. Means \pm SEM. ${ }^{\mathrm{a}} p<0.05 v s$. d6; ${ }^{\mathrm{b}} p<0.05$ vs. d6 and d10; ${ }^{*} p<0.05$ vs. control. 

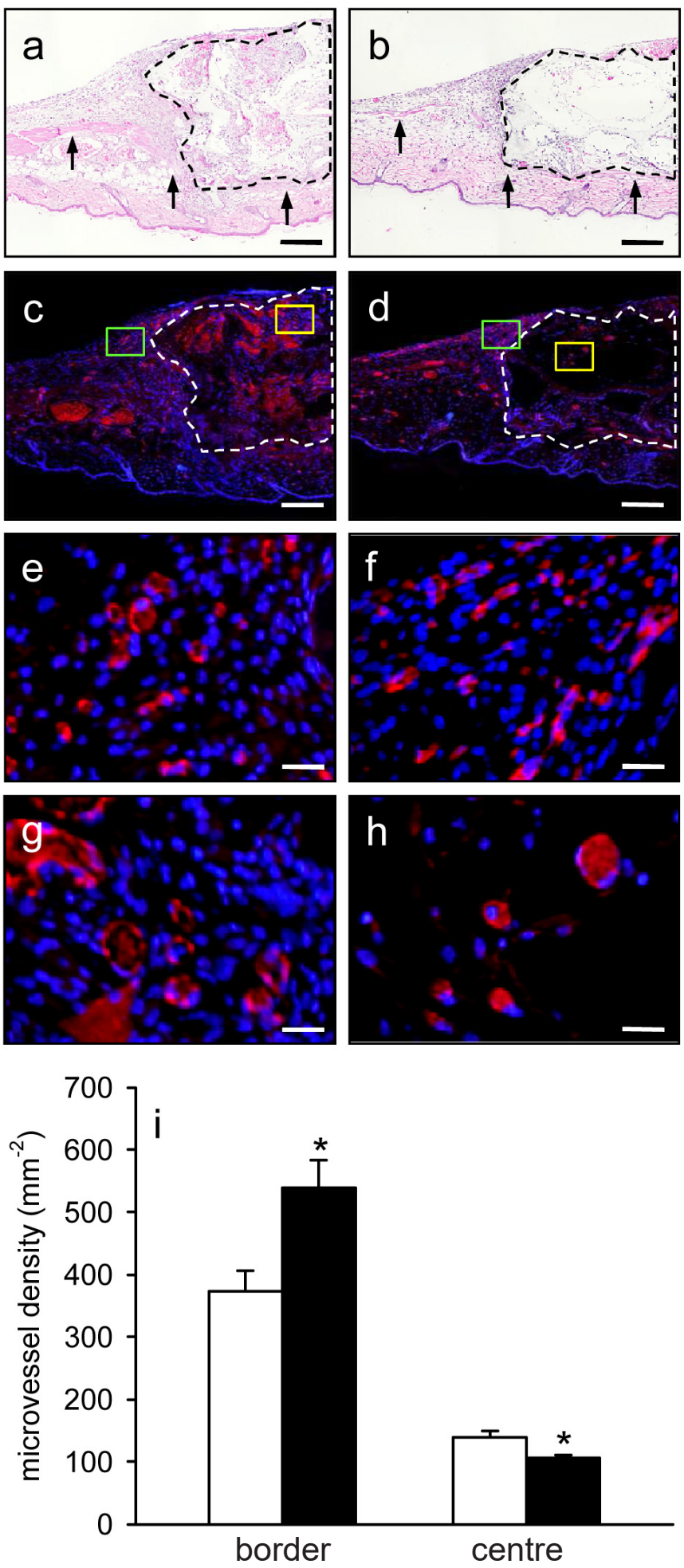

Fig. 5. (a,b) HE-stained sections of microvessel-seeded polyurethane scaffolds (borders marked by broken line) at day 14 after implantation onto the striated muscle tissue (arrows) of a vehicle-treated (a) and a MALP-2-treated (b) dorsal skinfold chamber. Note the less pronounced ingrowth of granulation tissue into the pores of the scaffold in the MALP2-treated chamber (compare b vs. a). Scale bars: $225 \mu \mathrm{m}$. (c-h) Immunohistochemical detection of CD31-positive microvessels (red) in the surrounding tissue (e,f = higher magnification of green rectangle in $\mathrm{c}$ and $\mathrm{d})$ and the centre $(\mathbf{g}$, $\mathbf{h}=$ higher magnification of yellow rectangle in $\mathbf{c}$ and $\mathbf{d}$ ) of microvessel-seeded polyurethane scaffolds (borders marked by broken line in $\mathbf{c}$ and $\mathbf{d}$ ) at day 14 after implantation into a vehicle-treated (c) and a MALP-2-treated (d) dorsal skinfold chamber. Note that the tissue surrounding the scaffold in the MALP-2-treated chamber (f) exhibits a higher microvessel density compared to the control (e). In contrast, the centre of the scaffold (h) contains less microvessels compared to the control (g). The sections are stained with Hoechst 33342 to identify cell nuclei (blue). Scale bars: c, d = $225 \mu \mathrm{m}$; $\mathbf{e}-\mathbf{h}=22 \mu \mathrm{m}$. (i) Microvessel density $\left(\mathrm{mm}^{-2}\right)$ in the border and centre of microvessel-seeded polyurethane scaffolds at day 14 after implantation into vehicle-treated (control; white bars, $n=7$ ) and MALP-2-treated (black bars, $n=7$ ) dorsal skinfold chambers, as assessed by immunohistochemistry. Means \pm SEM. ${ }^{*} p<0.05$ vs. control. 

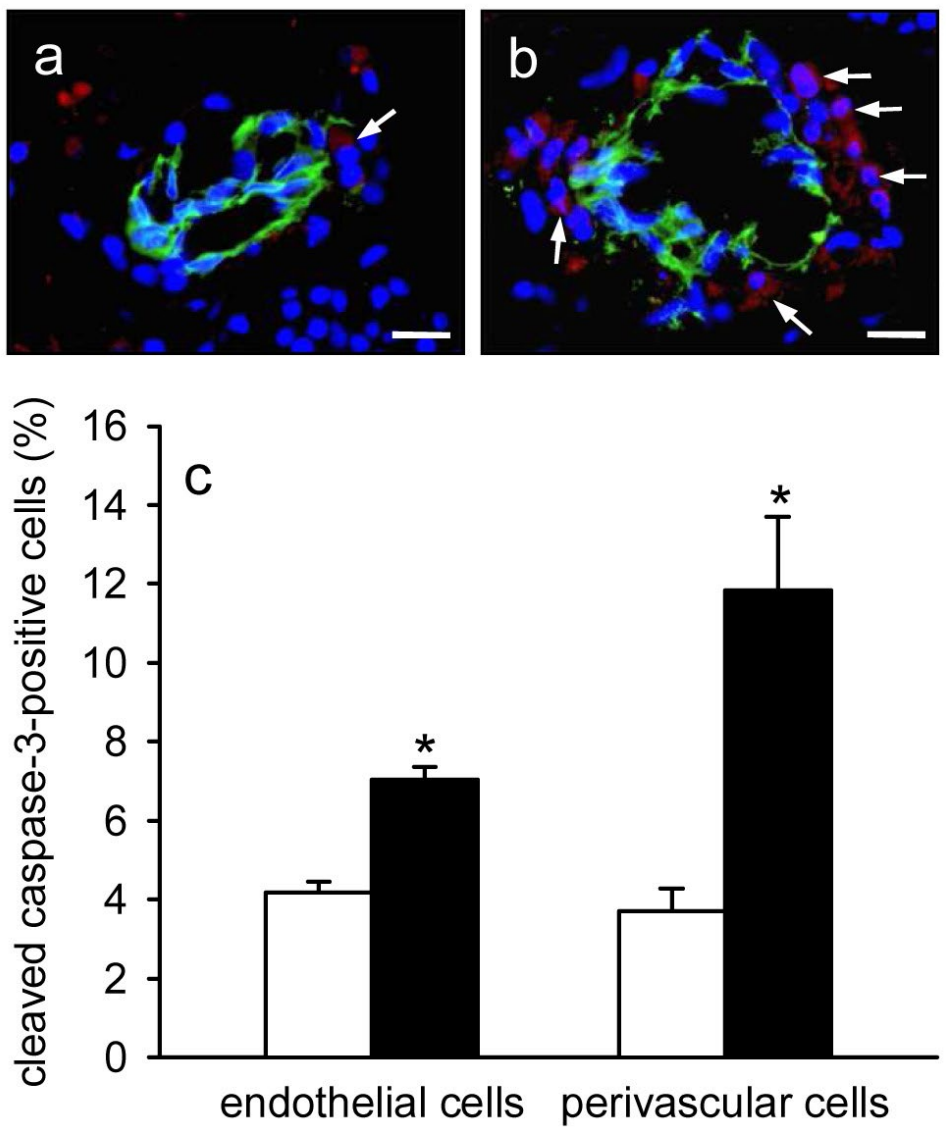

Fig. 6. (a,b) Immunohistochemical detection of cleaved caspase-3-positive cells (red) in microvascular fragments, which were cultured for $24 \mathrm{~h}$ in EC medium supplemented with vehicle (a) or MALP-2 (b). The sections were additionally stained with an antibody against CD31 for the detection of endothelial cells (green) and with Hoechst 33342 to identify cell nuclei (blue). Note that the MALP-2-treated microvascular fragment exhibits a higher number of cleaved caspase-3positive perivascular cells (b, arrows) when compared to the vehicle-treated control (a). Scale bars: $18 \mu \mathrm{m}$. (c) Cleaved caspase-3-positive endothelial cells and perivascular cells (\%) in microvascular fragments, which were cultured for $24 \mathrm{~h}$ in EC medium supplemented with vehicle (control; white bars, $n=3$ ) or MALP-2 (black bars, $n=3$ ), as assessed by immunohistochemistry. Means \pm SEM. ${ }^{*} p<0.05$ vs. control.

(Laschke et al., 2012). However, beside endothelial cells, microvascular fragments still exhibit a mixture of different perivascular cell types, including vessel wall-stabilising pericytes, endothelial progenitor cells and mesenchymal stem cells within their physiological niche (Laschke et al., 2012; McDaniel et al., 2014). In an additional set of in vitro experiments, we quantified the number of cleaved caspase-3-positive apoptotic endothelial and perivascular cells in isolated microvascular fragments, which had been cultured for $24 \mathrm{~h}$ in EC medium supplemented with MALP2 or vehicle. Of interest, we found that exposure to MALP2 significantly increased both the number of apoptotic endothelial and perivascular cells when compared to vehicle-treated controls (Fig. 6a-c).

\section{Discussion}

In the present study, we analysed the effects of MALP-2 on the vascularisation of polyurethane scaffolds seeded with adipose tissue-derived microvascular fragments. Previously, we could demonstrate that these fragments can be used as effective vascularisation units in tissue engineering, which develop into new microvascular networks within implanted scaffolds (Laschke et al., 2012; Laschke et al., 2014b). This process, however, takes several days. During this time period the cells inside the implants may suffer from critical hypoxia. On the other hand, we have recently shown that local application of MALP-2 is a promising strategy to improve the angiogenic host tissue response to implanted biomaterials (Laschke et al., 2014a). Hence, we herein speculated that this approach may accelerate and enhance the vascularisation of microvessel-seeded scaffolds. Unexpectedly, we found that despite an improved vascularisation at the implantation site exposure to MALP-2 impaired the development of new microvascular networks within the scaffolds.

By means of intravital fluorescence microscopy, we repetitively analysed leukocyte-endothelial cell interactions to characterise the inflammatory host tissue response in venules of MALP-2-treated and vehicle-treated dorsal skinfold chambers. In the two experimental groups, these venules exhibited comparable diameters, centreline $\mathrm{RBC}$ velocities and wall shear rates. This indicates that differences in leukocyte-endothelial cell interactions are not due to different micro-haemodynamic conditions. In 
line with our previous study (Laschke et al., 2014a), local application of MALP-2 induced a temporary inflammation of the host site, which was restricted to the first $3 \mathrm{~d}$ after scaffold implantation. This may have contributed to the improved vascularisation of the granulation tissue developing around the scaffolds during the 14-day implantation period. In fact, accumulation of leukocytes and macrophages at inflammatory sites is typically associated with the local release of pro-angiogenic growth factors, which stimulate the development of new blood vessels (David Dong et al., 2009; Kreuger and Phillipson, 2016). On the other hand, MALP-2 is not only a potent stimulator of the immune system, but has also been shown to exert direct angiogenic effects. (Grote et al., 2010) reported that TLR2/6-dependent stimulation of endothelial cells by MALP-2 promotes their proliferation, migration and tube forming capacity. They further found that these effects can also be induced by MALP-2-stimulated MSCs, which secrete high amounts of angiogenic growth factors, such as VEGF (Grote et al., 2013b). Accordingly, tissue-resident MSCs may support MALP-2-induced angiogenesis in a paracrine manner.

Microvascular fragments seeded on implanted scaffolds have to reassemble to new microvascular networks. They also have to develop interconnections to the microvessels of the surrounding host tissue for the onset of an own blood supply. These are typical angiogenic processes, which are characterised by sprout formation, vessel growth and vascular remodelling. However, despite its pro-angiogenic properties, MALP-2 did not promote these processes. In fact, we found that microvascular fragment-seeded scaffolds exposed to MALP-2 exhibited a significantly reduced number of perfused ROIs and a lower functional microvessel density when compared to vehicle-treated controls. This was associated with less tissue ingrowth into the implants. We additionally found that vessel maturation and vascular remodelling was markedly impaired in the implants, as indicated by a delayed diameter reduction and deteriorated blood perfusion of the newly developing microvascular networks (Vollmar et al., 2001). Accordingly, wall shear rates were reduced in these networks when compared to controls. This, in turn, may have contributed to a deteriorated scaffold vascularisation, because shear stress is well known to be a driving force of angiogenesis (Brown and Hudlicka, 2003; Resnick et al., 2003).

To unravel the mechanisms underlying the detrimental effects of MALP-2 on microvascular fragments, we additionally analysed the viability of isolated microvascular fragments, which had been cultured for $24 \mathrm{~h}$ in EC medium supplemented with MALP-2 or vehicle. We could demonstrate that MALP-2-treated microvascular fragments exhibit a significantly increased number of apoptotic endothelial and perivascular cells when compared to vehicle-treated controls. This observation is in line with the findings of (Into et al., 2004). They reported that MALP2 activates nuclear factor (NF)- $\mathrm{\kappa B}$ in cultured HEK293 cells, followed by $\mathrm{p} 38$ mitogen activated protein kinase (MAPK)-mediated apoptosis. Hence, we suggest that this pro-apoptotic effect of MALP-2 may have markedly affected the vascularisation capacity of microvascular fragments in our in vivo experiments. Of interest, this was not associated with the induction of apoptotic cell death in the host microvasculature, as indicated by an improved vascularisation of the granulation tissue surrounding the MALP-2-treated implants. These discrepant observations may be explained by the fact that the microvascular fragments underwent an enzymatic isolation procedure and suffered from hypoxia in the initial phase after scaffold implantation. In addition, it is well known that microvascular blood flow and shear forces are essential for endothelial cell homeostasis (Chien 2007; Weber et al., 2010). Cessation of blood flow, as it is the case in freshly isolated microvascular fragments, promotes endothelial cell apoptosis (Meeson et al., 1996). Taken together, these additional stresses may have substantially increased the susceptibility of the microvascular fragments to the pro-apoptotic MALP-2 stimulus when compared to the host microvessels, which were protected within their physiological environment.

\section{Conclusions}

We could demonstrate in the present study that local treatment of the implantation site with MALP-2 improves the angiogenic host tissue response to polyurethane scaffolds. However, MALP-2 treatment impaired the capacity of adipose tissue-derived microvascular fragments to develop into new microvascular networks within the implants. Accordingly, the herein evaluated approach may not be recommended to further improve the vascularisation capacity of microvascular fragments in tissue engineering applications.

\section{Acknowledgements}

This work was supported by the Large Bone Defect Healing Program of the AO Foundation. We are grateful for the excellent technical assistance of Janine Becker and Ruth M. Nickels.

\section{References}

Auger FA, Gibot L, Lacroix D (2013) The pivotal role of vascularisation in tissue engineering. Annu Rev Biomed Eng 15: 177-200.

Baatz H, Steinbauer M, Harris AG, Krombach F (1995) Kinetics of white blood cell staining by intravascular administration of rhodamine 6G. Int J Microcirc Clin Exp 15: 85-91.

Brown MD, Hudlicka O (2003) Modulation of physiological angiogenesis in skeletal muscle by mechanical forces: involvement of VEGF and metalloproteinases. Angiogenesis 6: 1-14.

Chien S (2007) Mechanotransduction and endothelial cell homeostasis: the wisdom of the cell. Am J Physiol Heart Circ Physiol 292: H1209-H1224.

David Dong ZM, Aplin AC, Nicosia RF (2009) Regulation of angiogenesis by macrophages, dendritic 
cells, and circulating myelomonocytic cells. Curr Pharm Des 15: 365-379.

Debergh I, Pattyn P, Ceelen W (2015) Microvascular effects of the low molecular weight heparins in a colorectal xenograft model: an intravital microscopy study. J Surg Res 194: 488-495.

Deiters U, Gumenscheimer M, Galanos C, Mühlradt PF (2003) Toll-like receptor 2- and 6-mediated stimulation by macrophage-activating lipopeptide 2 induces lipopolysaccharide (LPS) cross tolerance in mice, which results in protection from tumor necrosis factor alpha but in only partial protection from lethal LPS doses. Infect Immun 71: 4456-4462.

Grote K, Schuett H, Salguero G, Grothusen C, Jagielska J, Drexler H, Mühlradt PF, Schieffer B (2010) Toll-like receptor 2/6 stimulation promotes angiogenesis via GMCSF as a potential strategy for immune defense and tissue regeneration. Blood 115: 2543-2552.

Grote K, Sonnenschein K, Kapopara PR, Hillmer A, Grothusen C, Salguero G, Kotlarz D, Schuett H, Bavendiek U, Schieffer B (2013a) Toll-like receptor 2/6 agonist macrophage-activating lipopeptide- 2 promotes reendothelialization and inhibits neointima formation after vascular injury. Arterioscler Thromb Vasc Biol 33: 2097 2104.

Grote K, Petri M, Liu C, Jehn P, Spalthoff S, Kokemüller H, Luchtefeld M, Tschernig T, Krettek C, Haasper C, Jagodzinski M (2013b) Toll-like receptor 2/6-dependent stimulation of mesenchymal stem cells promotes angiogenesis by paracrine factors. Eur Cell Mater 26: 66-79.

Herr N, Mauler M, Bode C, Duerschmied D (2015) Intravital Microscopy of Leukocyte-endothelial and Platelet-leukocyte Interactions in Mesenterial Veins in Mice. J Vis Exp 102: e53077.

Hiscox AM, Stone AL, Limesand S, Hoying JB, Williams SK (2008) An islet-stabilizing implant constructed using a preformed vasculature. Tissue Eng Part A 14: 433 440.

Hoffmann JN, Vollmar B, Laschke MW, Inthorn D, Fertmann J, Schildberg FW, Menger MD (2004) Microhemodynamic and cellular mechanisms of activated protein $\mathrm{C}$ action during endotoxemia. Crit Care Med 32: 1011-1017.

Hoying JB, Boswell CA, Williams SK (1996) Angiogenic potential of microvessel fragments established in three-dimensional collagen gels. In Vitro Cell Dev Biol Anim 32: 409-419.

Into T, Kiura K, Yasuda M, Kataoka H, Inoue N, Hasebe A, Takeda K, Akira S, Shibata K (2004) Stimulation of human Toll-like receptor (TLR) 2 and TLR6 with membrane lipoproteins of Mycoplasma fermentans induces apoptotic cell death after NF-kappa B activation. Cell Microbiol 6: 187-199.

Kremer T, Abé D, Weihrauch M, Peters C, Gebhardt MM, Germann G, Heitmann C, Walther A (2008) Burn plasma transfer induces burn edema in healthy rats. Shock 30: 394-400.

Kreuger J, Phillipson M (2016) Targeting vascular and leukocyte communication in angiogenesis, inflammation and fibrosis. Nat Rev Drug Discov 15: 125-142.
Laschke MW, Strohe A, Menger MD, Alini M, Eglin D (2010) In vitro and in vivo evaluation of a novel nanosize hydroxyapatite particles/poly(ester-urethane) composite scaffold for bone tissue engineering. Acta Biomater 6: 2020-2027.

Laschke MW, Vollmar B, Menger MD (2011) The dorsal skinfold chamber: window into the dynamic interaction of biomaterials with their surrounding host tissue. Eur Cell Mater 22: 147-164.

Laschke MW, Kleer S, Scheuer C, Schuler S, Garcia P, Eglin D, Alini M, Menger MD (2012) Vascularisation of porous scaffolds is improved by incorporation of adipose tissue-derived microvascular fragments. Eur Cell Mater 24: 266-277.

Laschke MW, Schank TE, Scheuer C, Kleer S, Schuler S, Metzger W, Eglin D, Alini M, Menger MD (2013) Threedimensional spheroids of adipose-derived mesenchymal stem cells are potent initiators of blood vessel formation in porous polyurethane scaffolds. Acta Biomater 9: 68766884.

Laschke MW, Augustin V, Kleer S, Tschernig T, Menger MD (2014a) Locally applied macrophage-activating lipopeptide-2 (MALP-2) promotes early vascularisation of implanted porous polyethylene $\left(\right.$ Medpor $\left.^{\circledR}\right)$. Acta Biomater 10: 4661-4669.

Laschke MW, Grässer C, Kleer S, Scheuer C, Eglin D, Alini M, Menger MD (2014b) Adipose tissue-derived microvascular fragments from aged donors exhibit an impaired vascularisation capacity. Eur Cell Mater 28: 287-298.

Laschke MW, Kleer S, Scheuer C, Eglin D, Alini M, Menger MD (2015) Pre-cultivation of adipose tissuederived microvascular fragments in porous scaffolds does not improve their in vivo vascularisation potential. Eur Cell Mater 29: 190-200.

Lührmann A, Tschernig T, Pabst R, Niewiesk S (2005) Improved intranasal immunization with live-attenuated measles virus after co-inoculation of the lipopeptide MALP-2. Vaccine 23: 4721-4726.

McDaniel JS, Pilia M, Ward CL, Pollot BE, Rathbone CR (2014) Characterization and multilineage potential of cells derived from isolated microvascular fragments. J Surg Res 192: 214-222.

Meeson A, Palmer M, Calfon M, Lang R (1996) A relationship between apoptosis and flow during programmed capillary regression is revealed by vital analysis. Development 122: 3929-3938.

Mühlradt PF, Kiess M, Meyer H, Süssmuth R, Jung G (1997) Isolation, structure elucidation, and synthesis of a macrophage stimulatory lipopeptide from Mycoplasma fermentans acting at picomolar concentration. J Exp Med 185: 1951-1958.

Niebuhr M, Mühlradt PF, Wittmann M, Kapp A, Werfel $T$ (2008) Intracutaneous injection of the macrophageactivating lipopeptide-2 (MALP-2) which accelerates wound healing in mice - a phase I trial in 12 patients. Exp Dermatol 17: 1052-1056.

Novosel EC, Kleinhans C, Kluger PJ (2011) Vascularisation is the key challenge in tissue engineering. Adv Drug Deliv Rev 63: 300-311. 
Nunes SS, Krishnan L, Gerard CS, Dale JR, Maddie MA, Benton RL, Hoying JB (2010) Angiogenic potential of microvessel fragments is independent of the tissue of origin and can be influenced by the cellular composition of the implants. Microcirculation 17: 557-567.

Pilia M, McDaniel JS, Guda T, Chen XK, Rhoads RP, Allen RE, Corona BT, Rathbone CR (2014) Transplantation and perfusion of microvascular fragments in a rodent model of volumetric muscle loss injury. Eur Cell Mater 28: 11-23.

Resnick N, Yahav H, Shay-Salit A, Shushy M, Schubert S, Zilberman LC, Wofovitz E (2003) Fluid shear stress and the vascular endothelium: for better and for worse. Prog Biophys Mol Biol 81: 177-199.

Rharbaoui F, Drabner B, Borsutzky S, Winckler U, Morr M, Ensoli B, Mühlradt PF, Guzmán CA (2002) The Mycoplasma-derived lipopeptide MALP-2 is a potent mucosal adjuvant. Eur J Immunol 32: 2857-2865.

Rouwkema J, Khademhosseini A(2016) Vascularisation and angiogenesis in tissue engineering: Beyond creating static networks. Trends Biotechnol doi: 10.1016/j. tibtech.2016.03.002.

Rücker M, Laschke MW, Junker D, Carvalho C, Schramm A, Mülhaupt R, Gellrich NC, Menger MD (2006) Angiogenic and inflammatory response to biodegradable scaffolds in dorsal skinfold chambers of mice. Biomaterials 27: 5027-5038.

Schmidt J, Welsch T, Jäger D, Mühlradt PF, Büchler MW, Märten A (2007) Intratumoural injection of the toll-like receptor-2/6 agonist 'macrophage-activating lipopeptide-2' in patients with pancreatic carcinoma: a phase I/II trial. Br J Cancer 97: 598-604.

Shepherd BR, Chen HY, Smith CM, Gruionu G, Williams SK, Hoying JB (2004) Rapid perfusion and network remodeling in a microvascular construct after implantation. Arterioscler Thromb Vasc Biol 24: 898-904.

Vollmar B, Laschke MW, Rohan R, Koenig J, Menger MD (2001) In vivo imaging of physiological angiogenesis from immature to preovulatory ovarian follicles. Am J Pathol 159: 1661-1670.

Weber M, Baker MB, Moore JP, Searles CD (2010) MiR-21 is induced in endothelial cells by shear stress and modulates apoptosis and eNOS activity. Biochem Biophys Res Commun 393: 643-648.

\section{Discussion with Reviewer}

Arnaud Scherberich: The study is rather iterative, since it presents no novel concept as compared to previous studies from the same group, but just a technical improvement of engraftment of the grafts by the use of MALP-2.

Authors: The reviewer is correct that we have already demonstrated that i) the seeding of scaffolds with microvascular fragments (Laschke et al., 2012) and ii) the local stimulation of the implantation site with MALP-2 (Laschke et al., 2014a) represent promising vascularisation strategies for tissue engineering and regenerative medicine. However, for exactly this reason, we hypothesised in the present study that the previously observed beneficial effects of the two approaches may be ideally potentiated by the combination of both. We feel that this is a comprehensible objective for the study. In addition, the unexpected findings of the present study are of particular importance because they indicate that the combination of the two approaches cannot be recommended to further improve the vascularisation capacity of microvascular fragments in tissue engineering applications.

Arnaud Scherberich: Wouldn't the testing of human adipose microvascular fragments in mice be more relevant than mice ones? Were microvascular fragments from both species already compared in vivo? Do they share a similar biology/functionality?

Authors: We agree that it would be highly interesting to test microvascular fragments of human origin in mice and to compare their biology/functionality with murine microvascular fragments. To our knowledge this has not been done so far. However, for such a type of analysis, the human microvascular fragments would have needed to be transplanted into immune-incompetent animals to prevent host tissue rejection. This, in turn, may have markedly influenced the results of our study, because many effects of MALP-2 are mediated by immunological processes, as stated in the Introduction section of our manuscript. Therefore, we feel that it would be much better to compare human and murine microvascular fragments in a separate study independent of the MALP-2 focus of the present study.

Petra Kluger: The set-up of an animal model is very time and cost intensive. There is already existing data confirming the beneficial effect of MALP-2 on vascular ingrowth (Laschke et al., 2014a), however, another mentioned study demonstrated adverse effects of MALP-2 (Into et al, 2004). To exclude a general negative impact on the complementary target cells, the analysis of a possible apoptotic effect of MALP-2 would have been required prior to the in vivo investigation.

Authors: We agree with the reviewer that it is reasonable for time, cost and ethical reasons to analyse initially the effects of a compound in vitro and only in a second step in vivo. However, based on our previous in vivo results (Laschke et al., 2014a), we were convinced that MALP-2 exerts a positive effect on the vascularisation capacity of microvascular fragments. Hence, we decided to directly perform an animal experiment. Only after the unexpected result that MALP-2 markedly impairs the development of new microvascular networks within the vessel-seeded scaffolds, we searched for a possible explanation. Therefore, we herein performed elaborate in vivo investigations prior to the in vitro analyses, which is also the reason for the order of the presented results.

Editor's note: The Scientific Editor responsible for this paper was Juerg Gasser. 